\title{
Electromagnetic Leakage Testing System Based on Labwindows/CVI
}

\author{
Yingzhu Chen", a Yuwen Wang 2, b , Hanjing $\mathrm{Li}^{2, \mathrm{c}}$ and Qiang Shü, d \\ ${ }^{1}$ University of electronic, science and technology of China, Chengdu 611731, China; \\ 2University of electronic, science and technology of China, Chengdu 611731, China. \\ a1147133516@qq.com, b83211668@qq.com, c448063460@qq.com, d874611955@qq.com
}

Keywords: electromagnetic leakage, spectrum monitoring, software development.

\begin{abstract}
An electromagnetic leakage testing system was designed in this paper based on Lab Windows/CVI. The system combined antenna and spectrum analyzer to test the electromagnetic leakage of the computer system.
\end{abstract}

\section{Introduction}

With the development of the information society, the use of computer systems is becoming more and more extensive. When the computer is working, it will radiate electromagnetic waves to the outside world [1]. The two main modes are radiation coupling and conduction coupling [2]. These electromagnetic waves contain information that the computer is dealing with, and once received by other devices, it will lead to electromagnetic leakage $[3,4]$. Therefore, the electromagnetic leakage test system is designed in this paper to test the electromagnetic leakage of the computer system.

\section{Design of Electromagnetic Leakage Test System}

\subsection{The Structure of the Electromagnetic Leakage Testing System.}

The electromagnetic leakage testing system designed in this paper is composed of antenna, spectrum analyzer and computer. Its structure is shown in the following figure.

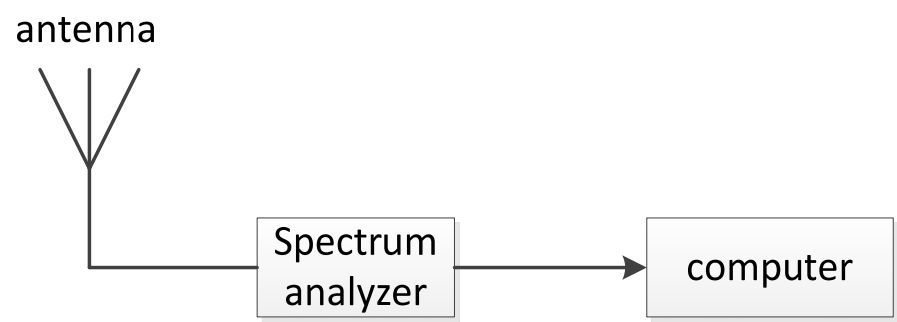

Fig 1. the structure of the electromagnetic leakage testing system

As is shown in Fig.1, the antenna is used to receive the electromagnetic leakage signals and the spectrum analyzer is used to measure the intensity of electromagnetic leakage signal. The computer is used to install the electromagnetic leakage software. This paper connects the spectrum analyzer and the computer with the net wire.

\subsection{Noise Analysis of Electromagnetic Leakage Test System.}

The noise in the electromagnetic leakage test system is mainly divided into the external environment noise and the internal noise of the spectrum analyzer. The external environment noise can be suppressed by means of shielding. And the internal noise of the spectrum analyzer is mainly white noise. A method for measuring the signal to noise ratio of the leakage signal of a computer system is proposed [5].

The section headings are in boldface capital and lowercase letters. Second level headings are typed as part of the succeeding paragraph (like the subsection heading of this paragraph). All manuscripts must be in English, also the table and figure texts, otherwise we cannot publish your paper. Please keep a second copy of your manuscript in your office. When receiving the paper, we assume that the corresponding authors grant us the copyright to use the paper for the book or journal in question. When receiving the paper, we assume that the corresponding authors grant us the copyright to use the 
paper for the book or journal in question. When receiving the paper, we assume that the corresponding authors grant us the copyright to use. When testing the background environment signal, the set received by the spectrum analyzer is $\mathrm{B}$, and the intensity of the background noise can be expressed as follows.

$$
B=10 \log N
$$

When testing the leakage signal, if the value of the signal received by the spectrum analyzer is A, the strength of the signal that belongs to the pure leakage signal is $\mathrm{S}$, then the intensity of the received noise leakage signal can be expressed as follows.

$$
A=10 \log (N+S)
$$

So, the signal to noise ratio of the leak signal is expressed as follows.

$$
S N R=\frac{S}{N}=\frac{10^{\frac{A}{10}}-10^{\frac{B}{10}}}{10^{\frac{B}{10}}}=10^{\frac{A-B}{10}}-1
$$

Conversion to the $\mathrm{dB}$ dimension is as follows.

$$
(S N R)_{d B}=10 \log \left(10^{\frac{A-B}{10}}-1\right)
$$

So, when the leakage signal strength is far greater than the background noise intensity, leakage signal and background noise signal to noise ratio is approximated as follows.

$$
(S N R)_{d B}=A-B
$$

We use the above formula to estimate the signal to noise ratio of the leakage signal.

\subsection{Denoising Algorithm for Electromagnetic Leakage Signal.}

According to the previous analysis, the noise in the electromagnetic leakage signal is mainly white noise. We use the new wavelet threshold function de-noising method proposed in literature [6] to denoise the leakage signal. Firstly, the sampling signal is transformed according to the selected good wavelet. Then the wavelet coefficients according to a selected threshold comparison decision to eliminate the noise of wavelet coefficients. Finally, the wavelet coefficients of the signal are replaced by the wavelet transform, and the reconstructed signal will no longer contain noise.

\subsection{Software Development of Electromagnetic Leakage Test System.}

The software structure of the electromagnetic leakage test system designed in this paper is as follows:

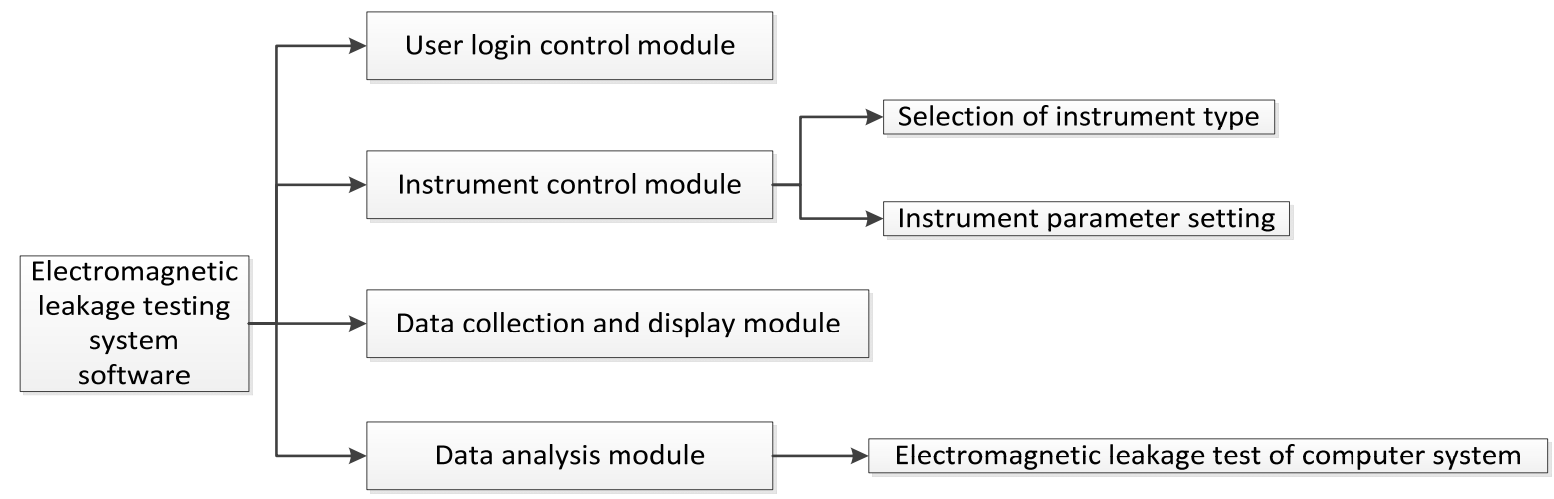

Fig 2. the structure of the electromagnetic leakage testing system software

As is shown in Fig.2, the electromagnetic leakage testing system software is composed of User login control module, instrument control module, data collection and display module and data analysis module. The user login and control module are used to identify the identity of a tester for software manipulation. We have set three sets of account names and passwords to log the system in advance as follows. 
Table 1. Login user table

\begin{tabular}{cccc}
\hline Numble & User name & Password & Jurisdiction \\
\hline 1 & UESTC & UESTC203 & Administrators \\
2 & admin & admin & Ordinary users \\
3 & peter & abcdefg & Ordinary users \\
\hline
\end{tabular}

Among them, only the user 'UESTC' can manage the user, and other users can only log in to the system for testing. The user name and password need to be entered in front of the user's main boundary, and the system is withdrawn from the three-consecutive error.

The instrument control module completes the control of the spectrum analyzer by the computer. The software of the electromagnetic leakage test system designed in this paper adopts the LAN interface, which can realize the selection of Aotewei9020, Aotewei9040 and AngilentE440X series spectrum analyzer.

The data collection and display module collect the data measured by spectrum analyzer and repaint the signal waveform.

The data analysis module completes the extraction and analysis of the leakage signals of the collected computer system components. Based on the Lab Window/CVI software development platform, we use the $\mathrm{C}$ language to carry out the electromagnetic leakage testing system software.

The flow chart of the electromagnetic leakage test system is shown as follows:

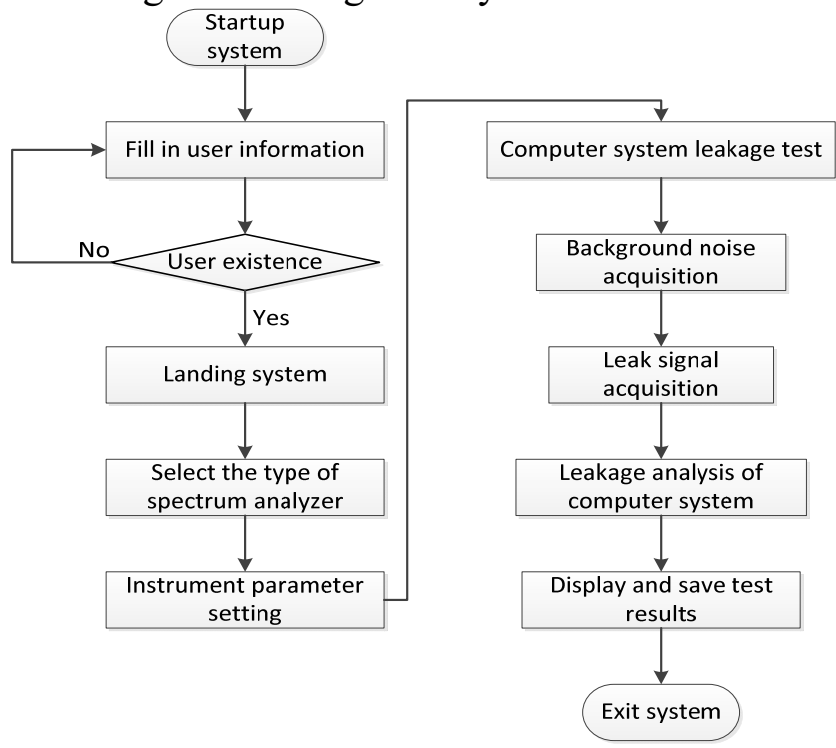

Fig 3. the flow chart of the electromagnetic leakage test system

\subsection{Test of Electromagnetic Leakage Test System.}

The software interface of the electromagnetic leakage test system designed in this paper is shown in Figure 4.

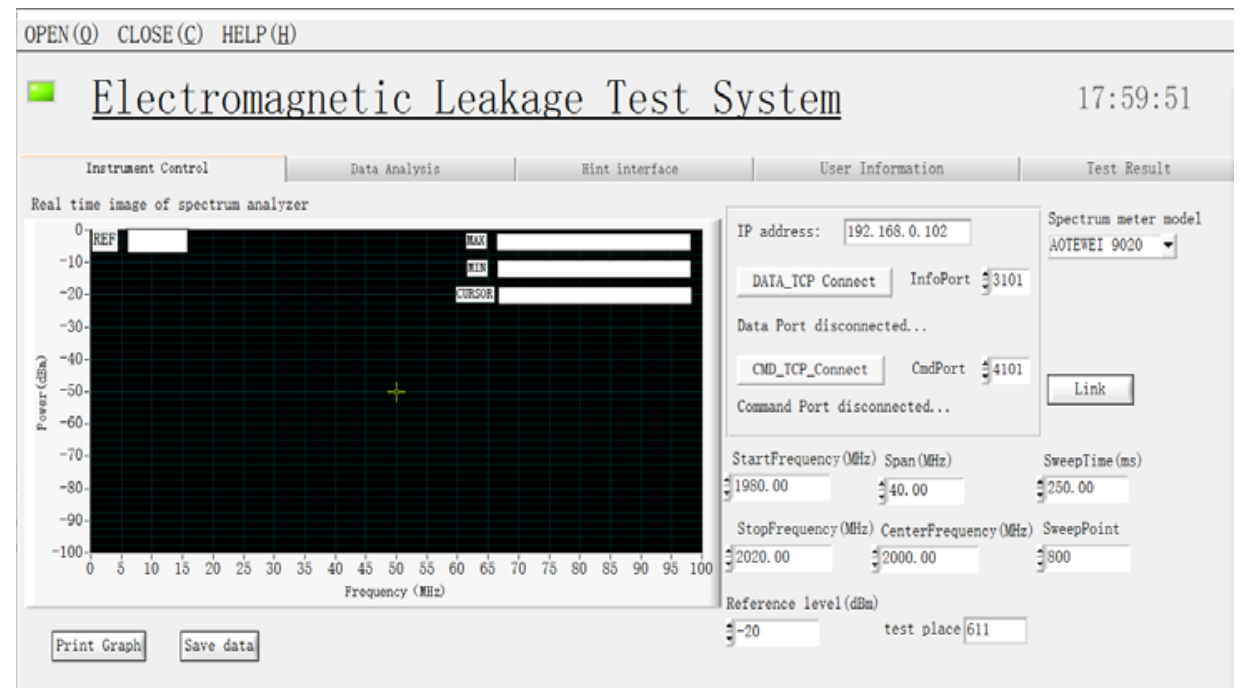

Fig 4. Main interface of electromagnetic leakage test system 
Now we adjust the frequency range to $300 \mathrm{MHz}$ and place the measuring antenna at one meter away from the host computer to test the electromagnetic leakage information of it. First turn off the host computer and use the spectrum analyzer to measure the background noise data. Then turn on the host computer and test the noise leakage data. The background noise data and the noise leakage data are shown in Fig.5 and Fig.6.

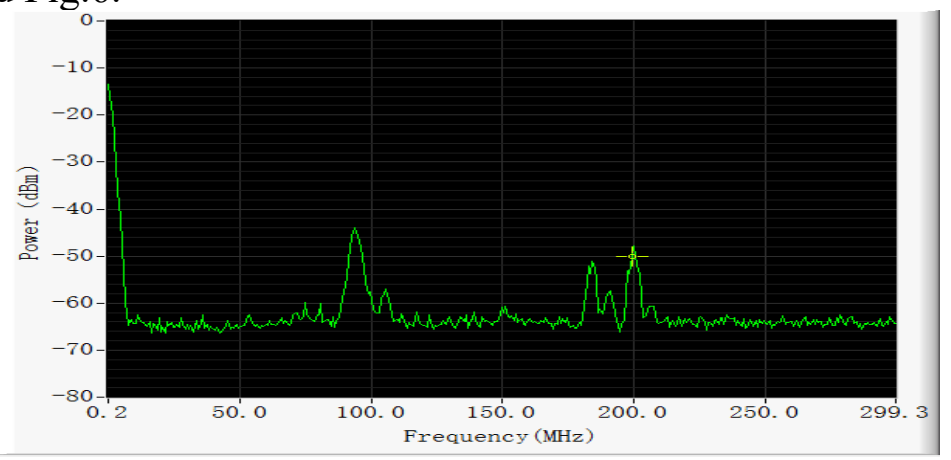

Fig 5. the background noise data

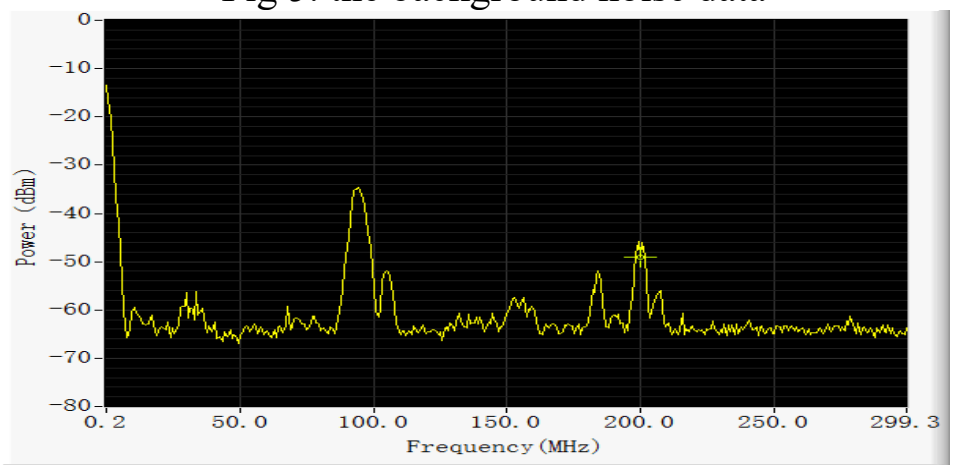

Fig 6. the noise leakage data

By clicking the wavelet denoising button to denoise and subtract the above two sets of signals we can obtain the electromagnetic leakage signal shown in Fig.6.

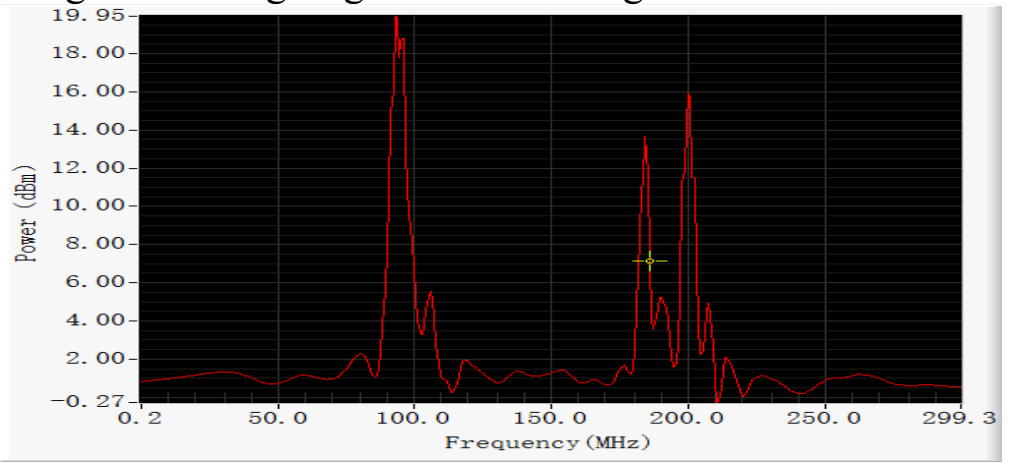

Fig 7. the electromagnetic leakage signal

As is shown in Fig.6, the maximum leakage point of the computer display is at $94 \mathrm{MHz}$ and the the upper leakage frequency of the host computer is $200 \mathrm{MHz}$. The intensity of the leakage signal of the maximum leakage frequency is $19.95 \mathrm{dBm}$.

\section{Summary}

According to the leakage test experiment of the computer host, we can see that the software of the electromagnetic leakage testing system designed in this paper can meet the leak testing function of the computer system.

\section{References}

[1]. Yu Y, Deng Y. Research of computer electromagnetic information leakage based on three layers of protection model[J]. Journal of Chemical \& Pharmaceutical Research, 2014. 
[2]. Lu L, Nie Y, Zhang $\mathrm{H}$. The electromagnetic leakage and protection for computer[C]. International Symposium on Electromagnetic Compatibility Proceedings. IEEE, 1997:378-382.

[3]. Jiang H. Electromagnetic Leakage Protection Technology[J]. Computer Engineering \& Software, 2011.

[4]. Guo H. Electromagnetic Leakage Analysis of RF Simulation System[J]. Ship Electronic Engineering, 2011.

[5]. Shi sen. The red and black signal analysis of the electromagnetic leakage of computer host [D]., Taiyuan University of Science and Technology, 2011.

[6]. Chen Yingzhu, Wang Yuwen, Yang Wei, et al. A new threshold function wavelet de-noising algorithm for [J]. communication technology, 2017, 50 (7): 1407-1411. 